\title{
Komorbiditäten beachten!
}

\section{Rheuma kommt selten allein...}

\begin{abstract}
Rheumatiker sterben heute meist nicht an ihrer Erkrankung, sondern an Komorbiditäten wie Herzinfarkt oder Schlaganfall.
\end{abstract}

$80 \%$ der Rheumapatienten haben mindestens eine, mehrere oder viele Komorbiditäten. Diese Zahl hat das Deutsche Rheuma-Forschungszentrum (DRFZ) in Berlin ermittelt. Einige davon bestimmen heute die Prognose der Erkrankung.

\section{Kardiales Risiko nahezu verdoppelt}

Nicht mehr die Krankheit selber, sondern kardiovaskuläre und pulmonale Komorbiditäten sind die wichtigste Todesursache bei Rheumapatienten. So ist das Risiko für MACE (Major Adverse Cardiovascular Events) bei Patienten mit hochaktiver RA im Vergleich zur Normalbevölkerung nahezu verdoppelt [1]. Ein erhöhtes Risiko zeigt sich auch bei anderen entzündlich-rheumatischen Erkrankungen, ist aber bei RA besonders hoch.

\section{Verpflichtet nach Komorbiditäten zu suchen!}

Die hohe kardiovaskuläre Morbidität und Mortalität ist primär der chronischen systemischen Entzündung bei rheumatischen Erkrankungen geschuldet. Gemeinsam mit traditionellen Risikofaktoren wie Hypertonie und Dyslipidämie triggert sie die rasche AtheroskleroseProgression. Zudem spielen die eingesetzten Medikamente eine ambivalente Rolle, manche tragen zu einer Erhöhung des kardiovaskulären Risikos bei [2]. So haben NSAR zum Beispiel ein relatives Risiko für Herzinfarkt von 1,13, Glukokortikoide - insbesondere bei Langzeitanwendung - von 1,41. Methotrexat und vor allem TNF- $\alpha$-Hemmer senken das Risiko primär durch die anhaltende Verringerung der Krankheitsaktivität.

Wie wichtig es ist, bei Rheumapatienten nach Komorbiditäten zu suchen, zeigt eine Studie aus Dänemark [3]. Hier hat

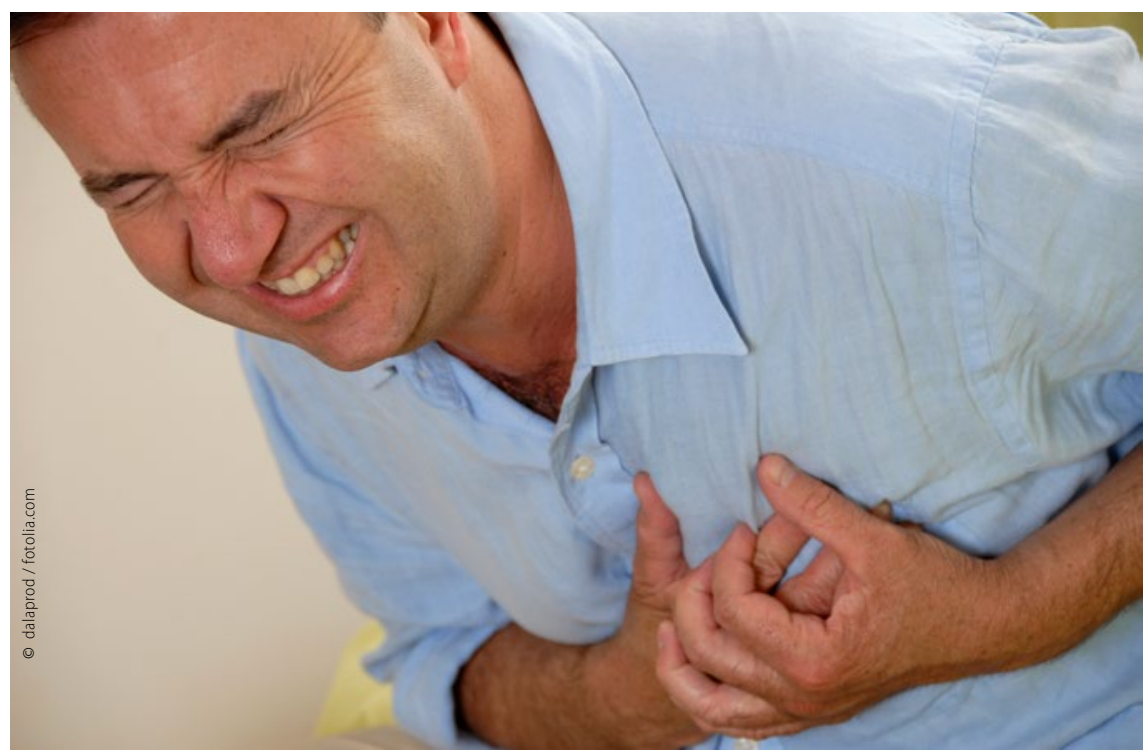

Gerade das Herz gilt es bei Patienten mit rheumatischen Erkrankungen besonders gut im Auge zu behalten.

man bei 644 herzgesunden Rheumapatienten nach kardiovaskulären Risikofaktoren gesucht. Bei 36\% wurde eine bis dahin nicht bekannte Hypertonie gefunden, bei über $55 \%$ war das LDL-Cholesterin erhöht ( $\geq 3,0 \mathrm{mM} / \mathrm{l})$, bei $14 \%$ der Nüchternblutzucker. Bei der Anwendung eines pathologischen Risikoscores, des EuroScores, hatten $20 \%$ ein hohes Risiko für fatale kardiovaskuläre Ereignisse.

„Wenn sie die Diagnose RA gestellt haben, sind Sie dazu verpflichtet, nach Komorbiditäten zu suchen“, appellierte der Rheumatologe Prof. Dr. Klaus Krüger vom Praxiszentrum St. Bonifatius in München. Die Erkennung und Minimierung von Risikofaktoren sei die gemeinsame Aufgabe des Rheumatologen und des Hausarztes, so Krüger beim PraxisUpdate in Wiesbaden.

\section{EULAR bietet Hilfestellung}

Weitere Komorbiditäten bei RA sind interstitielle Lungenerkrankungen. Sie sind nicht ganz so häufig wie kardiovaskuläre Erkrankungen, haben aber eine extrem schlechte Prognose: Das Fünf-JahresÜberleben liegt zwischen 40-50\%. Bis zu $30 \%$ der RA-Patienten leiden an einer Depression, die häufig übersehen wird, weil der Bewegungsapparat im Vordergrund steht. Weitere Komorbiditäten sind $\mathrm{zu}$ etwa $22 \%$ Osteoporose, bis zu $60 \%$ Anämie, und bis zu 90\% der Patienten leiden unter einer Fatigue.

Dem Thema Komorbiditäten galt auch beim diesjährigen Kongress der European League Against Rheumatism (EULAR) in Rom ein besonderes Augenmerk. Als Komorbiditäten, die es im Auge zu behalten gelte, listet die EULAR auch maligne Erkrankungen und peptische Ulzera. Geplant ist ein Core Set, um die standardisierte Erfassung von Komorbiditäten $\mathrm{zu}$ erleichtern. Dieses kann in den nächsten Tagen von der Website heruntergeladen werden.

(Christina Ott)

[1] Ann Rheum Dis 2015, 74, 326-332

[2] Ann Rheum Dis 2015, 74, 326-332

[3] Ann Rheum Dis 2013; 72: 1771-1776

[4] Ann Rheum Dis 2015; 74 (2): 9 